\title{
LARGE-TIME STABILITY OF TRAVELLING WAVES FOR A CLASS OF FULLY NONLINEAR PARABOLIC EQUATIONS
}

BY

FABIO CAMILLI (Dipartimento di Energetica, Facoltà di Ingegneria, Università degli Studi di L'Aquila, Monteluco di Roio, 67040 L'Aquila, Italy)

AND

MANUELA MOLINARI (Dipartimento di Matematica, Università degli Studi di Torino, Via Carlo Alberto, 10-10123 Torino, Italy)

Abstract. In this paper we prove existence and $L^{1}$ stability of travelling waves for a class of second-order nonlinear parabolic equations in divergence form. As a consequence of the previous result, we get stability in the $L^{\infty}$ norm of travelling waves for a class of fully nonlinear second-order equations.

1. Introduction. In this paper we investigate the asymptotic stability of travelling wave solutions to the equation

$$
v_{t}+F\left(v, v_{x}\right)_{x}=0
$$

where $v=v(x, t), x \in \mathbb{R}, t>0$ and $F: \mathbb{R} \times \mathbb{R} \rightarrow \mathbb{R}$ is twice continuously differentiable and satisfies

(H1) $F_{M}(p, M) \leq-\alpha$ for some $\alpha>0$, for any $(p, M) \in \mathbb{R} \times \mathbb{R}$;

(H2) $F_{M M}(p, M) \geq 0$ for any $(p, M) \in \mathbb{R} \times \mathbb{R}$.

A large literature has been devoted to the study of stability for scalar viscous conservation laws, i.e., for $F(p, M)=f(p)-M$ (see, for example, [IO58, GJK93, MN94, Ser99] and the references therein).

Concerning results in the $L^{1}$ norm, the first one was obtained by Osher and Ralston [OR82]. Imposing the restriction that the initial data is a perturbation of the travelling wave that does not exceed its profile, they proved that the corresponding solution of the viscous conservation law converges in the $L^{1}$ norm to the travelling wave as $t \rightarrow+\infty$. The proof is based on the semigroup properties of the equation. The same result was later proved by Serre [Ser99] in generalized form.

In [FS98] the restriction on the perturbation of the initial data made in [OR82] was removed. The key step here is to prove the stability in the case of a constant travelling

Received October 3, 2000.

2000 Mathematics Subject Classification. Primary 35K55, 35B35.

E-mail address: camilli@ing.univaq.it

E-mail address: molinari@dm.unito.it 
wave. Then this result, together with the semigroup properties of the equation, allows us to control the behaviour of the part of the initial data exceeding the profile of the travelling wave.

Recently, using a similar technique, some stability results for a system of conservation laws coming from relaxation have been obtained in [MN96, Ser98].

The aim of this paper is to extend the result of [OR82], [FS98] to the case of Eq. (1.1). We will adopt the technique used in [Ser99, FS98].

We first prove existence of a travelling wave solution to (1.1), making use of conditions analogous to the Rankine-Hugoniot condition and the Oleinik entropy condition for scalar conservation laws. Then (Sec. 2) we prove stability of the asymptotic profile under the Osher and Ralston condition on the initial data. The final step is to remove the restriction on the initial data and this will be done in Sec. 3. In this part a crucial role is played by hypothesis (H2), which allows us to control the nonlinear second-order term.

In Sec. 4, employing the well-known relation between conservation laws and HamiltonJacobi equations, we show that the $L^{1}$ stability of travelling waves for conservation laws is translated into $L^{\infty}$ stability of travelling waves for fully nonlinear equations of the type

$$
u_{t}+F\left(u_{x}, u_{x x}\right)=0
$$

In the appendix we prove a lemma that allows us to control the number of zeros of a solution of Eq. (1.1).

Acknowledgments. The authors would like to thank Professor P. Marcati for having suggested to them this problem and for his continuous support.

2. Stability under Osher-Ralston assumption. Equation (1.1) generates a semigroup as in the case of viscous conservation laws. For an initial data $v_{0}(x)$ we denote by $S(t) v_{0}(x)$ the solution to (1.1) at time $t$. Then $\left\{S(t): L^{\infty}(\mathbb{R}) \rightarrow L^{\infty}(\mathbb{R}) ; t \geq 0\right\}$ is a semigroup satisfying the following properties:

(P1) (Regularity). If $v_{0} \in L^{\infty}(\mathbb{R})$, then $S(t) v_{0}(x)$ is regular for all $t>0$.

(P2) (Comparison). If $v_{0} \leq u_{0}$ a.e., then $S(t) v_{0} \leq S(t) u_{0}$ a.e. for all $t>0$.

(P3) (Conservation). If $v_{0}-u_{0} \in L^{1}(\mathbb{R})$, then for all $t>0, S(t) v_{0}-S(t) u_{0} \in L^{1}(\mathbb{R})$ and $\int_{-\infty}^{+\infty}\left\{S(t) v_{0}-S(t) u_{0}\right\} d x=\int_{-\infty}^{+\infty}\left\{v_{0}-u_{0}\right\} d x$.

(P4) (Contraction). If $v_{0}-u_{0} \in L^{1}(\mathbb{R})$, then $\left\|S(t) v_{0}-S(t) u_{0}\right\|_{L^{1}(\mathbb{R})} \leq\left\|v_{0}-u_{0}\right\|_{L^{1}(\mathbb{R})}$ for all $t>0$.

While (P2)-(P4) are easily obtained employing the divergence structure of the equation, (P1) comes from regularity results for fully nonlinear second-order equations (see, for example, [VH69, Don91, Wan92]). Now we study existence of a travelling wave, i.e., of a solution of the type $\varphi(x, t)=\varphi(x-s t)$, for $(1.1)$

Proposition 2.1. Let $v^{+} \neq v^{-}$and $v^{+}<v^{-}$. Set

$$
s=\frac{F\left(v^{+}, 0\right)-F\left(v^{-}, 0\right)}{v^{+}-v^{-}}
$$


and assume that $F$ satisfies

$$
s<\frac{F(v, 0)-F\left(v^{-}, 0\right)}{v-v^{-}} \text {for all } v \in\left(v^{+}, v^{-}\right) .
$$

Then there exists a (unique up to a translation) travelling wave solution $\varphi$ of (1.1) satisfying $\varphi( \pm \infty)=v^{ \pm}$, with speed $s$ given by $(2.1)$.

Proof. We look for a solution of $(1.1)$ in the form $v(x, t)=\varphi(x-s t)$. Then we get the ordinary differential equation for the function $\varphi$,

$$
-s \varphi^{\prime}+F\left(\varphi, \varphi^{\prime}\right)^{\prime}=0
$$

Integrating the previous equation between $-\infty$ and $x$, we obtain

$$
-s \varphi+F\left(\varphi, \varphi^{\prime}\right)=q
$$

where

$$
q=-s v^{-}+F\left(v^{-}, 0\right) .
$$

Passing to the limit as $x \rightarrow+\infty$ in (2.3) we have

$$
-s v^{+}+F\left(v^{+}, 0\right)=q
$$

that, together with (2.4), gives (2.1). Existence and uniqueness of a local solution to Eq. (2.3) follows by (H1). Conditions (H1) and (2.2) imply that this solution is strictly decreasing. Finally, (2.4), (2.5) give the conditions at infinity $\varphi( \pm \infty)=v^{ \pm}$.

REMARK 2.2. Conditions (2.1) and (2.2) can be seen as a generalization of the Rankine-Hugoniot and, respectively, of the Oleinik entropy condition for fully nonlinear equations.

Let $v$ be the solution of (1.1) with an initial data $v_{0}$ that does not exceed the profile of the travelling wave. We will show that the $\omega$-limit set of $v(x, t)$ is attractive in $L^{1}$ and reduces to a single element that is a translation of the travelling wave.

THEOREM 2.3. Let $\varphi$ be a travelling wave solution of $(1.1)$ with $\varphi( \pm \infty)=v^{ \pm}\left(v^{+} \neq v^{-}\right.$ and $\left.v^{+}<v^{-}\right)$. Then for any function $v_{0} \in \varphi+\psi, \psi \in L^{1}(\mathbb{R})$, such that

$$
v_{0}(x) \in[\inf \varphi, \sup \varphi] \text { for all } x \in \mathbb{R},
$$

the solution $v$ of (1.1) with initial data $v_{0}$ satisfies

$$
\lim _{t \rightarrow+\infty}\|v(\cdot, t)-\varphi(\cdot-s t+\delta)\|_{L^{1}(\mathbb{R})}=0
$$

where

$$
\delta=\frac{\int_{-\infty}^{+\infty}\left\{v_{0}(x)-\varphi(x)\right\} d x}{v^{+}-v^{-}} .
$$

Proof. We assume that $s=0$, i.e., $\varphi(x, t)=\varphi(x)$, since we can always reduce to this case by changing variables.

We first observe (see Lemma 2.4) that it is not restrictive to assume the initial data to be in between two translations of the travelling wave, i.e.,

$$
\varphi(x+\alpha) \leq v_{0}(x) \leq \varphi(x+\beta) \quad \text { for all } x \in \mathbb{R}
$$

for some $\alpha, \beta \in \mathbb{R}$. 
Hence, the comparison property (P2) implies

$$
\varphi(x+\alpha) \leq v(x, t) \leq \varphi(x+\beta) \quad \text { for all } x \in \mathbb{R}, t \geq 0 .
$$

Define

$$
w(x, t)=v(x, t)-\varphi(x)
$$

Then

$$
\varphi(x+\alpha)-\varphi(x) \leq w(x, t) \leq \varphi(x+\beta)-\varphi(x) .
$$

Since $\varphi(\cdot+\alpha)-\varphi(\cdot), \varphi(\cdot+\beta)-\varphi(\cdot) \in L^{1}(\mathbb{R}),\|w(\cdot, t)\|_{L^{1}(\mathbb{R})}$ is uniformly bounded for any $t \geq 0$. Moreover, by the contraction property (P4), it follows that

$$
\begin{aligned}
\|w(\cdot+h, t)\|_{L^{1}(\mathbb{R})} \leq & \|v(\cdot+h, t)-v(\cdot, t)\|_{L^{1}(\mathbb{R})}+\|\varphi(\cdot+h)-\varphi(\cdot)\|_{L^{1}(\mathbb{R})} \\
\leq & \left\|v_{0}(\cdot+h)-v_{0}(\cdot)\right\|_{L^{1}(\mathbb{R})}+\|\varphi(\cdot+h)-\varphi(\cdot)\|_{L^{1}(\mathbb{R})} \\
\leq & \|\varphi(\cdot+h)-\varphi(\cdot)\|_{L^{1}(\mathbb{R})}+\|\psi(\cdot+h)-\psi(\cdot)\|_{L^{1}(\mathbb{R})} \\
& +\|\varphi(\cdot+h)-\varphi(\cdot)\|_{L^{1}(\mathbb{R})} \rightarrow 0 \quad \text { as } h \rightarrow 0 .
\end{aligned}
$$

Therefore $\{w(\cdot, t)\}_{t>0}$ is also equicontinuous in $L^{1}$ and the Riesz-Fréchet-Kolmogorov theorem [Bre83] implies that it is relatively compact in $L^{1}(\mathbb{R})$. Denote by $B_{s}$ the set of accumulation points of $\{w(\cdot, t)\}_{t>s}$. By compactness, $B_{s}$ is not empty and, since

$$
\mathcal{A}=\varphi(x)+\bigcap_{s \geq 0} B_{s}
$$

is the intersection of a decreasing family of not empty compact sets, it is not empty.

The set $\mathcal{A}$ is invariant under the semigroup $S$, since if $a \in \mathcal{A}$, then $a=\lim _{n \rightarrow+\infty} v\left(t_{n}\right)$ implies that $S(t) a=\lim _{n \rightarrow+\infty} S(t) v\left(t_{n}\right)=\lim _{n \rightarrow+\infty} v\left(t+t_{n}\right) \in \mathcal{A}$. Finally, the semigroup $S$ is surjective on $\mathcal{A}$ and, by the regularity property (P1), the elements of $\mathcal{A}$ are regular functions.

For any $k \in \mathbb{R}$ fixed, the function

$$
t \mapsto\|v(\cdot, t)-\varphi(\cdot-k)\|_{L^{1}(\mathbb{R})}
$$

is decreasing because of (P4). Therefore, it admits a limit $c(k)$ for $t \rightarrow+\infty$. Take $a \in \mathcal{A}$ and let $\left\{t_{n}\right\}$ be such that

$$
\lim _{n \rightarrow+\infty}\left\|v\left(\cdot, t_{n}\right)-a(\cdot)\right\|_{L^{1}(\mathbb{R})}=0 .
$$

Then (2.8) and (2.9) imply

$$
\|a(\cdot)-\varphi(\cdot-k)\|_{L^{1}(\mathbb{R})}=c(k) .
$$

We claim that

$$
a\left(x_{0}\right)=\varphi\left(x_{0}-k\right) \text { implies } a^{\prime}\left(x_{0}\right)=\varphi^{\prime}\left(x_{0}-k\right) .
$$

Assume that the claim is true. Let $k(x)$ be a function such that $a(x)=\varphi(x-k(x))$ for any $x \in \mathbb{R}$. Since $\varphi$ is strictly decreasing and smooth and $a(x)$ is smooth, then $k(x)$ is well defined and smooth. By construction, $a(x)=\varphi(x-k(x))$ and by $(2.11)$, we have

$$
\varphi^{\prime}(x-k(x)) k^{\prime}(x)=0
$$


Then $k^{\prime}(x)=0$ for any $x \in \mathbb{R}$ and therefore

$$
a(x)=\varphi(x-k),
$$

i.e., the attractor reduces to $\{\varphi(x-k)\}$. The final step is to show that $k=-\delta$ with $\delta$ given by (2.7). By the conservation property (P3) we have

$$
0=\int_{\mathbb{R}} \lim _{t \rightarrow+\infty}\left\{v\left(x, t_{n}\right)-v_{0}(x)\right\} d x=\int_{\mathbb{R}}\left\{\varphi(x-k)-v_{0}(x)\right\} d x,
$$

and therefore,

$$
\int_{\mathbb{R}}\{\varphi(x-k)-\varphi(x)\} d x=-\int_{\mathbb{R}}\left\{\varphi(x)-v_{0}(x)\right\} d x .
$$

On the other side,

$$
\int_{\mathbb{R}}\{\varphi(x-k)-\varphi(x)\} d x=-k\left(v^{+}-v^{-}\right)
$$

which, together with (2.12), implies

$$
k=\frac{\int_{-\infty}^{+\infty}\left\{v_{0}(x)-\varphi(x)\right\} d x}{v^{+}-v^{-}}=-\delta .
$$

Since the family $\{w(t)\}_{t \geq 0}$ is relatively compact in $L^{1}(\mathbb{R})$, it converges as $t \rightarrow+\infty$ and therefore

$$
\lim _{t \rightarrow+\infty}\|v(\cdot, t)-\varphi(\cdot+\delta)\|_{L^{1}(\mathbb{R})}=0 .
$$

To complete the proof of the theorem, we have to show the claim (2.11). Set

$$
\begin{aligned}
& z=S(t) a-\varphi(\cdot-k), \\
& u=S(t) a .
\end{aligned}
$$

Since $\varphi$ verifies Eq. (1.1), we have

$$
z_{t}+\left\{F\left(u, u_{x}\right)-F\left(\varphi, \varphi_{x}\right)\right\}_{x}=0 .
$$

Multiplying the previous equation by $\operatorname{sgn} z$ and integrating with respect to $x$, we get

$$
\frac{d}{d t} \int_{\mathbb{R}}|z(x, t)| d x=-\int_{\mathbb{R}}\left\{F\left(u, u_{x}\right)-F\left(\varphi, \varphi_{x}\right)\right\}_{x} \operatorname{sgn} z d x .
$$

Recalling (2.10) and since $\mathcal{A}$ is invariant, we have $\|z\|_{L^{1}(\mathbb{R})}=\|S(t) a-\varphi(\cdot-k)\|_{L^{1}(\mathbb{R})}$ is constant and therefore

$$
-\int_{\mathbb{R}}\left\{F\left(u, u_{x}\right)-F\left(\varphi, \varphi_{x}\right)\right\}_{x} \operatorname{sgn} z d x=0 .
$$

Set $J_{\varepsilon}(\tau)=\sqrt{\varepsilon^{2}+\tau^{2}}$. From (2.13) it follows that

$$
\begin{aligned}
0= & \lim _{\varepsilon \rightarrow 0} \int_{\mathbb{R}}\left\{F\left(u, u_{x}\right)-F\left(\varphi, \varphi_{x}\right)\right\}_{x} J_{\varepsilon}^{\prime}(z) d x \\
= & \lim _{\varepsilon \rightarrow 0} \int_{\mathbb{R}}\left\{F\left(u, \varphi_{x}\right)-F\left(\varphi, \varphi_{x}\right)\right\}_{x} J_{\varepsilon}^{\prime}(z) d x \\
& +\lim _{\varepsilon \rightarrow 0} \int_{\mathbb{R}}\left\{F\left(u, u_{x}\right)-F\left(u, \varphi_{x}\right)\right\}_{x} J_{\varepsilon}^{\prime}(z) d x .
\end{aligned}
$$


The first term in the previous equation vanishes. Indeed,

$$
\begin{aligned}
& \lim _{\varepsilon \rightarrow 0} \int_{\mathbb{R}}\left\{F\left(u, \varphi_{x}\right)-F\left(\varphi, \varphi_{x}\right)\right\}_{x} J_{\varepsilon}^{\prime}(z) d x \\
&=\lim _{\varepsilon \rightarrow 0} \int_{\{u \neq \varphi\}}\left(\int_{0}^{1} F_{p}\left(\vartheta u+(1-\vartheta) \varphi, \varphi_{x}\right) d \vartheta\right) z z_{x} J_{\varepsilon}^{\prime \prime}(z) d x .
\end{aligned}
$$

Observing that $|z| J_{\varepsilon}^{\prime \prime}(z) \rightarrow 0$ as $\varepsilon \rightarrow 0$ a.e. on $\{|z|>0\}=\{u \neq \varphi\}$, by the dominated convergence theorem we get

$$
\lim _{\varepsilon \rightarrow 0} \int_{\mathbb{R}}\left\{F\left(u, \varphi_{x}\right)-F\left(\varphi, \varphi_{x}\right)\right\}_{x} J_{\varepsilon}^{\prime}(z) d x=0 .
$$

Then integrating by parts the second term in (2.14), we get

$$
\lim _{\varepsilon \rightarrow 0} \int_{\mathbb{R}}\left\{F\left(u, u_{x}\right)-F\left(u, \varphi_{x}\right)\right\} z_{x} J_{\varepsilon}^{\prime \prime}(z) d x=0 .
$$

Let $x_{0}$ be such that $z\left(x_{0}\right)=0$ and take $\gamma>0$ in such a way that $|z|<\varepsilon$ in $\left(x_{0}-\gamma \varepsilon, x_{0}+\right.$ $\gamma \varepsilon)$. Since $J_{\varepsilon}^{\prime \prime}(\tau)=\frac{1}{\varepsilon} j\left(\frac{\tau}{\varepsilon}\right)$, where $j(\tau)=\left(1+\tau^{2}\right)^{-3 / 2}$, using (H2), we obtain

$$
\begin{aligned}
& \int_{\mathbb{R}}\left\{F\left(u, u_{x}\right)-F\left(u, \varphi_{x}\right)\right\} z_{x} J_{\varepsilon}^{\prime \prime}(z) d x \\
& \quad \leq-\alpha \int_{\mathbb{R}}\left|z_{x}\right|^{2} J_{\varepsilon}^{\prime \prime}(z) d x \\
& \quad \leq-\alpha \int_{x_{0}-\gamma \varepsilon}^{x_{0}+\gamma \varepsilon}\left|z_{x}\right|^{2} J_{\varepsilon}^{\prime \prime}(z) d x \\
& \quad \leq-\frac{\alpha}{\varepsilon} \int_{x_{0}-\gamma \varepsilon}^{x_{0}+\gamma \varepsilon}\left|z_{x}\right|^{2} j(1) d x \rightarrow-2 \gamma \alpha j(1) z_{x}\left(x_{0}\right) \quad \text { as } \varepsilon \rightarrow 0 .
\end{aligned}
$$

Hence from (2.15) it follows that

$$
z_{x}\left(x_{0}\right)=0 \text {. }
$$

In this way, we have proved that for all $a \in \mathcal{A}$ and for all $k \in \mathbb{R}$,

$$
z\left(x_{0}\right)=0 \text { implies } z_{x}\left(x_{0}\right)=0 .
$$

Taking $t=0$ in the previous formula we get the claim.

LEMMA 2.4. In order to prove Theorem 2.3, it is sufficient to assume that there exist $\alpha, \beta \in \mathbb{R}$ such that

$$
\varphi(x+\alpha) \leq v_{0}(x) \leq \varphi(x+\beta) \text { for all } x \in \mathbb{R} .
$$

Proof. A similar lemma has been proved in [MN96], and we sketch it here for the reader's convenience.

Let $v_{0}(x)=\varphi(x)+\psi(x)$ be the initial data, with $\psi \in L^{1}(\mathbb{R})$. By our assumption, $v_{0}(x) \in[\inf \varphi, \sup \varphi]$. Let $\left\{\psi_{n}\right\}_{n \in \mathbb{N}}$ be a sequence of functions such that $\left\{\psi_{n}\right\} \in C_{0}^{2}(\mathbb{R})$ and

$$
\lim _{n \rightarrow+\infty}\left\|\psi_{n}-\psi\right\|_{L^{1}(\mathbb{R})}=0
$$


Set $v_{0 n}=\varphi+\psi_{n}$ and denote by $v_{n}$ the solution of (1.1) with initial data $v_{0 n}$. Since $v_{0 n}$ satisfies (2.6), there exists a sequence $\left\{\delta_{n}\right\}_{n \in \mathbb{N}}$ such that

$$
\lim _{n \rightarrow+\infty}\left\|v_{n}(\cdot, t)-\psi\left(\cdot+\delta_{n}\right)\right\|_{L^{1}(\mathbb{R})}=0 .
$$

The sequence $\left\{\psi\left(\cdot+\delta_{n}\right)\right\}_{n \in \mathbb{N}}$ is a Cauchy sequence in $L^{1}(\mathbb{R})$. Indeed,

$$
\begin{aligned}
& \left\|\varphi\left(\cdot+\delta_{n}\right)-\varphi\left(\cdot+\delta_{m}\right)\right\|_{L^{1}(\mathbb{R})} \leq\left\|\varphi\left(\cdot+\delta_{n}\right)-v_{n}(\cdot, t)\right\|_{L^{1}(\mathbb{R})} \\
& \quad+\left\|v_{n}(\cdot, t)-v_{m}(\cdot, t)\right\|_{L^{1}(\mathbb{R})}+\left\|v_{m}(\cdot, t)-\varphi\left(\cdot+\delta_{m}\right)\right\|_{L^{1}(\mathbb{R})} \rightarrow 0 \quad \text { as } n \rightarrow+\infty
\end{aligned}
$$

from the contraction property (P4), (2.16) and (2.17). Since

$$
\int_{\mathbb{R}}\left\{\varphi\left(x+\delta_{n}\right)-\varphi\left(x+\delta_{m}\right)\right\} d x=\left(\delta_{n}-\delta_{m}\right)\left(v^{+}-v^{-}\right)
$$

(2.18) implies that $\left\{\delta_{n}\right\}$ converges, as $n \rightarrow+\infty$, to some limit $\delta$ and

$$
\lim _{n \rightarrow+\infty}\left\|\varphi\left(\cdot+\delta_{n}\right)-\varphi(\cdot+\delta)\right\|_{L^{1}(\mathbb{R})}=0 .
$$

Finally,

$$
\begin{aligned}
& \|v(\cdot, t)-\varphi(\cdot+\delta)\|_{L^{1}(\mathbb{R})} \leq\left\|v(\cdot, t)-v_{n}(\cdot, t)\right\|_{L^{1}(\mathbb{R})} \\
& \quad+\left\|v_{n}(\cdot, t)-\varphi\left(\cdot+\delta_{n}\right)\right\|_{L^{1}(\mathbb{R})}+\left\|\varphi\left(\cdot+\delta_{n}\right)-\varphi(\cdot+\delta)\right\|_{L^{1}(\mathbb{R})} \rightarrow 0 \quad \text { as } n \rightarrow+\infty
\end{aligned}
$$

3. The general result. This section is devoted to the proof of our main result.

ThEOREM 3.1. Let $\varphi$ be a travelling wave of $(1.1)$ with $\varphi( \pm \infty)=v^{ \pm}\left(v^{+} \neq v^{-}\right.$and $\left.v^{+}<v^{-}\right)$. Then for any function $v_{0} \in \varphi+L^{1}(\mathbb{R})$, the solution $v$ of (1.1) with initial data $v_{0}$ satisfies

$$
\lim _{t \rightarrow+\infty}\|v(\cdot, t)-\varphi(\cdot-s t+\delta)\|_{L^{1}(\mathbb{R})}=0,
$$

where $\delta$ is given as in (2.7).

We start stating a lemma that allows us to control the number of zeros of a solution of (1.1) and whose proof will be given in the Appendix. We denote with $\sharp \Omega$ the number of elements of $\Omega$.

LEMMA 3.2. Assume that $v_{0}(x) \in C^{1}(\mathbb{R})$ has only a finite number of zeros, say $M$, where it changes sign. If $v(x, t)$ is a solution of (1.1) with initial data $v_{0}$, then the number of zeros of $v(x, t)$ where it changes sign is bounded by $2 M$ for any $t>0$.

Following [FS98], we first prove Theorem 3.1 in the case of a constant state wave; namely, we suppose that the travelling wave $\varphi$ is a constant function $c$ (without loss of generality, we assume that $c=0$ ).

TheOREM 3.3. For any function $v_{0} \in L^{1}(\mathbb{R})$ such that

$$
\int_{\mathbb{R}} v_{0}(x) d x=0
$$


the solution $v$ of (1.1) with initial data $v_{0}$ satisfies

$$
\lim _{t \rightarrow+\infty}\|v(\cdot, t)\|_{L^{1}(\mathbb{R})}=0 .
$$

Proof. Thanks to the contraction property (P4), we can suppose that $v_{0} \in \mathcal{W}$, where $\mathcal{W}$ is the set of $C_{0}^{\infty}(\mathbb{R})$ functions having a finite number of zeros and where the function changes sign and satisfies $\left\|v_{0}\right\|_{L^{\infty}(\mathbb{R})}<r$ for $r>0$ and

$$
\left\|v_{0}\right\|_{L^{1}(\mathbb{R})} \sup _{[-r, r]}\left|F_{p}(p, 0)\right| \leq \alpha,
$$

where $\alpha$ is as in hypothesis (H1). We may also assume without loss of generality that $F(0,0)=F_{p}(0,0)=0$.

In order to estimate the $L^{1}$-norm of $v$, we consider the function $V$ defined by

$$
V(x, t)=\int_{-\infty}^{x} v(y, t) d y
$$

From the conservation property (P3) and (2.8), we get that $V(x, t) \rightarrow 0$ as $x \rightarrow \pm \infty$, for any $t>0$. Integrating Eq. (1.1) with respect to $x$, it follows that $V$ satisfies the equation

$$
V_{t}+F\left(V_{x}, V_{x x}\right)=0 .
$$

Expanding $F(p, M)$ with respect to the second variable at $\left(V_{x}, 0\right)$ and using the concavity assumption (H2), we get that

$$
V_{t}+F\left(V_{x}, 0\right)+F_{M}\left(V_{x}, 0\right) V_{x x} \leq 0
$$

for any $x \in \mathbb{R}, t>0$. Moreover, replacing $V$ by $V+\|V\|_{L^{\infty}(\mathbb{R})}$, we can assume that $V \geq 0$. Multiplying (3.3) by $V$ and integrating with respect to $x$, we get

$$
\begin{aligned}
\frac{1}{2} \frac{d}{d t} \int_{\mathbb{R}} V^{2}(x, t) d x & +\int_{\mathbb{R}} F\left(V_{x}(x, t), 0\right) V d x \\
& \leq-\int_{\mathbb{R}} F_{\Lambda I}\left(V_{x}(x, t), 0\right) V_{x x}(x, t) V(x, t) d x \leq-\alpha\|v(\cdot, t)\|_{L^{2}(\mathbb{R})}^{2},
\end{aligned}
$$

where we used that if $H(p)$ is a primitive of $F_{M}(p, 0)$, then by $(\mathrm{H} 1), H\left(V_{x}\right) V_{x} \leq-\alpha V_{x}^{2}$. Now to estimate the $L^{2}$-norm of $v$, we rewrite (1.1) as

$$
v_{t}+F(v, 0)_{x}+\left\{F\left(v, v_{x}\right)-F(v, 0)\right\}_{x}=0 .
$$

Multiplying by $v$, integrating with respect to $x$, and using the assumption (H1), we obtain

$$
\begin{aligned}
\frac{1}{2} \frac{d}{d t} \int_{\mathbb{R}} v^{2}(x, t) d x= & -\int_{\mathbb{R}} F(v(x, t), 0)_{x} v(x, t) d x \\
& -\int_{\mathbb{R}}\left\{F\left(v(x, t), v_{x}(x, t)\right)-F(v(x, t), 0)\right\}_{x} v(x, t) d x \\
= & \int_{\mathbb{R}}\left\{F\left(v(x, t), v_{x}(x, t)\right)-F(v(x, t), 0)\right\} v_{x}(x, t) d x \\
& =\int_{\mathbb{R}}\left\{\int_{0}^{1} F_{\Lambda}\left(v(x, t), \vartheta v_{x}(x, t)\right) d \vartheta v_{x}(x, t)\right\} v_{x}(x, t) d x \\
\leq & -\alpha \int_{\mathbb{R}} v_{x}^{2}(x, t) d x \leq 0,
\end{aligned}
$$


and therefore

$$
\frac{1}{2} \frac{d}{d t}\|v(\cdot, t)\|_{L^{2}(\mathbb{R})}^{2} \leq 0 .
$$

By the contraction property (P4),

$$
\|V(\cdot, t)\|_{L^{\infty}(\mathbb{R})} \leq\|v(\cdot, t)\|_{L^{1}(\mathbb{R})} \leq\left\|v_{0}\right\|_{L^{1}(\mathbb{R})}
$$

for all $t>0$. Since $\left\|v_{0}\right\|_{L^{1}(\mathbb{R})} \sup _{[-r, r]}\left|F_{p}(p, 0)\right| \leq \alpha$ for $\left\|v_{0}\right\|_{L^{\infty}(\mathbb{R})}<r$ by the assumptions, we have

$$
\begin{aligned}
\left|\int_{\mathbb{R}} F\left(V_{x}(x, t), 0\right) V(x, t) d x\right| & \leq\|V(\cdot, t)\|_{L^{\infty}(\mathbb{R})} \int_{\mathbb{R}}\left|F\left(V_{x}(x, t), 0\right)\right| d x \\
\leq & \frac{1}{2} \sup _{[-r, r]}\left|F_{p}(p, 0)\right|\left\|v_{0}\right\|_{L^{1}(\mathbb{R})} \int_{\mathbb{R}} v_{x}^{2}(x, t) d x \leq \frac{\alpha}{2} \int_{\mathbb{R}} v_{x}^{2}(x, t) d x .
\end{aligned}
$$

Then (3.4) and (3.6) imply

$$
\frac{1}{2} \frac{d}{d t}\|V(\cdot, t)\|_{L^{2}(\mathbb{R})}^{2} \leq-\frac{\alpha}{2}\|v(\cdot, t)\|_{L^{2}(\mathbb{R})}^{2} .
$$

Estimates (3.5) and (3.7) yield

$$
\lim _{t \rightarrow+\infty}\|v(\cdot, t)\|_{L^{2}(\mathbb{R})}^{2}=0
$$

and

$$
\|V(\cdot, t)\|_{L^{2}(\mathbb{R})} \leq\|V(\cdot, 0)\|_{L^{2}(\mathbb{R})} \quad \text { for all } t \geq 0
$$

Since

$$
V(x, t)^{2}=2 \int_{-\infty}^{x} v(y, t) V(y, t) d y \leq 2\|v(\cdot, t)\|_{L^{2}(\mathbb{R})}\|V(\cdot, t)\|_{L^{2}(\mathbb{R})},
$$

(3.8) and (3.9) imply

$$
\lim _{t \rightarrow+\infty}\|V(\cdot, t)\|_{L^{\infty}(\mathbb{R})}=0 .
$$

By Lemma 3.2, the number of zeros of $v(\cdot, t)$ where the function changes sign is uniformly bounded for all $t \geq 0$, by $2 M$, for some integer $M \geq 2$. Then there exist $2 M-1$ points $x_{1}, \ldots, x_{2 M-1}$ with $x_{0}=-\infty, x_{2 M}=+\infty$, such that $v(\cdot, t)$ has no change of sign on each of the intervals $\left[x_{i}, x_{i+1}\right], i=1, \ldots, 2 M-1$. Then

$$
\begin{aligned}
\|v(\cdot, t)\|_{L^{1}(\mathbb{R})} & =\int_{\mathbb{R}}|v(x, t)| d x=\sum_{i=0}^{2 M-1}\left|\int_{x_{i}}^{x_{i+1}} v(x, t) d x\right| \\
& =\sum_{i=0}^{2 M-1}\left|V\left(x_{i+1}, t\right)-V\left(x_{i}, t\right)\right| \\
& \leq 2(2 M-1)\|V(\cdot, t)\|_{L^{\infty}(\mathbb{R})} \rightarrow 0 \quad \text { as } t \rightarrow+\infty
\end{aligned}
$$

by $(3.10)$.

In the case of the viscous scalar conservation law, the proof that Theorem 3.1 follows from Theorem 2.3 and Theorem 3.3 is purely based on the semigroup properties satisfied by the equation. Since the same properties are satisfied by Eq. (1.1), the proof in [FS98] also applies to our case and we refer to it for the details. 
4. Stability for fully nonlinear equations. Let us consider the nonlinear secondorder parabolic equation

$$
u_{t}+F\left(u_{x}, u_{x x}\right)=0,
$$

where $F$ is a twice continuously differentiable function verifying the assumptions (H1), (H2). Examples of equations satisfying (H1), (H2) are the Hamilton-Jacobi-Bellman equation of control theory (but in this case $F$ is not regular), the following parabolic equation describing the evolution of a graph in the plane

$$
u_{t}+\frac{u_{x}^{2}}{1+u_{x}^{2}} u_{x x}-u_{x x}=0
$$

and an equation of the type

$$
u_{t}+u_{x}^{2}-\left(1+u_{x}^{2}\right) u_{x x}=0
$$

Other examples can be found in [Don91].

In this section, we will exploit the fact that if $u$ is a solution of (4.1), then $v=u_{x}$ is a solution of (1.1) to get stability results for perturbation of travelling waves of (4.1).

A function $\Phi(x, t)=\Phi(x-s t)$ that satisfies Eq. (4.1) is said to be a travelling wave for (4.1). The following proposition gives the existence of travelling wave solutions to (4.1).

Proposition 4.1. Let $u^{+}<0$. Define $s$ as in (2.1) and assume that $F$ satisfies (2.2). Then there exists a travelling wave solution $\Phi(x-s t)$ of $(4.1)$ such that $\Phi^{\prime}(-\infty)=0$, $\Phi^{\prime}(+\infty)=u^{+}$.

Proof. Differentiating (4.1) with respect to $x$ we get the equation

$$
v_{t}+F\left(v, v_{x}\right)_{x}=0 \text {. }
$$

Let $\varphi$ be the travelling wave for (4.2) given by Proposition 2.1 with $v^{-}=0, v^{+}=u^{+}$. Then $\varphi$ verifies

$$
-s \varphi+F\left(\varphi, \varphi^{\prime}\right)-F(0,0)=0
$$

Therefore, we have

$$
\begin{aligned}
-s \varphi & =F\left(\varphi, \varphi^{\prime}\right)-F\left(0, \varphi^{\prime}\right)+F\left(0, \varphi^{\prime}\right)-F(0,0) \\
& =F\left(\varphi, \varphi^{\prime}\right)-F\left(0, \varphi^{\prime}\right)+\int_{0}^{1} F_{M}\left(0, \vartheta \varphi^{\prime}\right) d \vartheta \varphi^{\prime} \\
& \leq F\left(\varphi, \varphi^{\prime}\right)-F\left(0, \varphi^{\prime}\right)-\alpha \varphi^{\prime},
\end{aligned}
$$

where $\alpha>0$ is given by assumption (H1). Then from (4.4) we get

$$
\varphi^{\prime} \leq \frac{1}{\alpha}\left\{\frac{F\left(\varphi, \varphi^{\prime}\right)-F\left(0, \varphi^{\prime}\right)}{\varphi}-s\right\} \varphi .
$$

Since $\varphi$ is decreasing and (4.3) holds, using (H1) we have

$$
-s \varphi+F\left(\varphi, \varphi^{\prime}\right)=F(0,0)<F\left(0, \varphi^{\prime}\right) .
$$

Therefore, from (4.5), for any $x_{0} \in \mathbb{R}$, there exists $\gamma>0$ such that

$$
\varphi^{\prime}(x) \leq \frac{1}{\alpha} \gamma \varphi \leq 0
$$


for any $x \in\left(-\infty, x_{0}\right)$. It follows that

$$
\begin{aligned}
\varphi^{+} & \leq \varphi(x) \leq \int_{-\infty}^{x-s t} \varphi^{\prime}(y) d y \\
& \leq \frac{1}{\alpha} \gamma \int_{-\infty}^{x-s t} \varphi(y) d y \leq 0 .
\end{aligned}
$$

Therefore, the function

$$
\Phi(x-s t)=\int_{-\infty}^{x-s t} \varphi(\xi) d \xi
$$

is well defined and satisfies

$$
-s \Phi^{\prime}+F\left(\Phi^{\prime}, \Phi^{\prime \prime}\right)=0
$$

with the conditions at infinity $\Phi^{\prime}(-\infty)=0, \Phi^{\prime}(+\infty)=u^{+}$. Hence $\Phi$ is a travelling wave for (4.1).

In the next theorem, we give the stability results for Eq. (4.1). The $L^{1}$ result for (1.1) translates into an $L^{\infty}$ result for its primitive.

Theorem 4.2. Let $\Phi$ be a travelling wave for (4.1). Then if $u_{0}=\Phi+\Psi$, where $\Psi$ is a continuous function with $\Psi^{\prime} \in L^{1}(\mathbb{R})$, the solution $u$ of (4.1) with initial data $u_{0}$ satisfies

$$
\lim _{t \rightarrow+\infty}\|u(\cdot, t)-\Phi(\cdot-s t+\delta)\|_{L^{\infty}(\mathbb{R})}=0
$$

where $\delta$ is given by (2.7) or equivalently by

$$
\delta=\frac{\left(u_{0}(+\infty)-u^{+}\right)-u_{0}(-\infty)}{u^{+}} .
$$

Proof. Let $\Phi$ be as in Proposition 4.1 and observe that the function $\Phi^{\prime}=\varphi$ is a travelling wave for $(4.2)$ satisfying $\varphi(-\infty)=0, \varphi(+\infty)=u^{+}$. Set $v_{0}(x)=u_{0}^{\prime}(x)=$ $\Phi^{\prime}+\psi^{\prime}$, and let $v$ be the solution of (4.2) with initial data $v_{0}$. Since $v_{0}$ satisfies the assumptions of Theorem 3.1, it follows that

$$
\lim _{t \rightarrow+\infty}\|v(\cdot, t)-\varphi(\cdot-s t+\delta)\|_{L^{1}(\mathbb{R})}=0 .
$$

Then

$$
u(x, t)=\int_{-\infty}^{x} v(y, t) d y
$$

and therefore we have

$$
\begin{aligned}
|u(x, t)-\Phi(x-s t+\delta)| & \leq\left|\int_{-\infty}^{x}\{v(y, t)-\varphi(y-s t+\delta)\} d y\right| \\
& \leq \int_{\mathbb{R}}|v(y, t)-\varphi(y-s t+\delta)| d y \rightarrow 0
\end{aligned}
$$

as $t \rightarrow+\infty$, for any $x \in \mathbb{R}$. 
5. Appendix. Denote by $\partial \Omega, \operatorname{Cl}(\Omega)$, and $\operatorname{Int}(\Omega)$, respectively, the topological boundary, the closure, and the interior of the set $\Omega$ and by $\sharp \Omega$ the number of elements of $\Omega$. Let $H_{n}$ be a smooth approximation of the Heaviside function in $L^{1}(\mathbb{R})$, such that

$$
H_{n}^{\prime} \rightarrow \delta_{0} \quad \text { in the sense of measure, }
$$

where $\delta_{0}$ denotes the usual Dirac unit mass.

We first quote the following lemma from [Mar96].

LEMMA 5.1. Let $f \in C^{1}(\mathbb{R})$ be such that

$$
\partial f^{-1}(0)=\left\{\alpha_{1}, \ldots, \alpha_{N}\right\}
$$

Denote by $\mathcal{N}=\left(f^{\prime}\right)^{-1}(0)$ and assume that

$$
\mathrm{Cl}(\operatorname{Int} \mathcal{N}) \cap \partial f^{-1}(0)=\varnothing .
$$

Then

$$
\liminf _{n \rightarrow+\infty} \int_{\mathbb{R}}\left|\frac{d}{d x} H_{n}(f(x))\right| d x \leq 2 N
$$

and

$$
\begin{aligned}
\int_{\mathbb{R}}\left|\frac{d}{d x} H(f(x))\right| d x & \leq \liminf _{n \rightarrow+\infty} \int_{\mathbb{R}}\left|\frac{d}{d x} H_{n}(f(x))\right| d x \\
& \leq 2 \int_{\mathbb{R}}\left|\frac{d}{d x} H_{n}(f(x))\right| d x .
\end{aligned}
$$

Moreover, if none of the zeros of $f$ is a minimum point, then

$$
\lim _{n \rightarrow+\infty} \int_{\mathbb{R}}\left|\frac{d}{d x} H_{n}(f(x))\right| d x=N .
$$

Proof of Lemma 3.2. Rewrite Eq. (1.1) in the form

$$
v_{t}+a(x, t) v_{x}+b(x, t) v_{x x}=0,
$$

where

$$
a(x, t)=F_{p}(v(x, t), 0)+\left(\int_{0}^{1} F_{M}\left(v(x, t), \vartheta v_{x}(x, t)\right) d \vartheta\right)_{x}
$$

and

$$
b(x, t)=\int_{0}^{1} F_{M}\left(v(x, t), \vartheta v_{x}(x, t)\right) d \vartheta
$$

Define

$$
H_{n}(x)=H_{1}(n x)
$$

with

$$
H_{1}(\lambda)=c \int_{0}^{\lambda} \exp \left\{-[y(1-y)]^{+}\right\}^{-1} d y,
$$

where the normalizing constant $c$ is chosen in such a way that $H_{1}(1)=1$. Multiplying (5.2) by $H_{n}^{\prime}(v)$, we have

$$
\left(H_{n}(v)\right)_{t}+a(x, t)\left(H_{n}(v)\right)_{x}+b(x, t) H_{n}^{\prime}(v) v_{x x}=0 .
$$


Since

$$
\begin{aligned}
{\left[b(x, t)\left(H_{n}(v)\right)_{x}\right]_{x}=} & b_{x}(x, t)\left(H_{n}(v)\right)_{x}+b(x, t)\left(H_{n}(v)\right)_{x x} \\
= & b_{x}(x, t)\left(H_{n}(v)\right)_{x}+b(x, t) H_{n}^{\prime \prime}(v) v_{x}^{2}+b H_{n}^{\prime}(v) v_{x x} \\
= & b_{x}(x, t)\left(H_{n}(v)\right)_{x}+b(x, t) \frac{H_{n}^{\prime \prime}(v)}{\left(H_{n}^{\prime}(v)\right)^{2}}\left(H_{n}(v)\right)_{x}^{2} \\
& +b(x, t) H_{n}^{\prime}(v) v_{x x},
\end{aligned}
$$

from (5.3) we get

$$
\begin{aligned}
\left(H_{n}(v)\right)_{t}+\left(a(x, t)-b_{x}(x, t)\right)\left(H_{n}(v)\right)_{x}+ & \left\{b(x, t)\left[H_{n}(v)\right]\right\}_{x} \\
& -b(x, t) H_{n}^{\prime \prime}(v)\left(H_{n}(v)\right)_{x}^{2}\left(H_{n}^{\prime}(v)\right)^{-2}=0 .
\end{aligned}
$$

Differentiating (5.4) with respect to $x$, setting $V_{n}=H_{n}(v)_{x}$ and multiplying the resulting equation by $\operatorname{sgn} V_{n}$, we obtain

$$
\begin{aligned}
\left|V_{n}\right|_{t}+ & \left\{\left(a(x, t)-b_{x}(x, t)\right)\left|V_{n}\right|\right\}_{x}+\left(a(x, t)-b_{x}(x, t)\right)\left|V_{n}\right| \operatorname{sgn}\left(V_{n}\right)_{x} \\
+\left(b(x, t)\left|V_{n}\right|\right)_{x x}-b(x, t)\left\{V_{n}\left(\operatorname{sgn} V_{n}\right)_{x}\right\}_{x} & -\left\{b(x, t)\left|V_{n}\right| V_{n} H_{n}^{\prime \prime}\left(H_{n}^{\prime}\right)^{-2}\right\}_{x} \\
& -b(x, t)\left|V_{n}\right| V_{n} H_{n}^{\prime \prime}\left(H_{n}^{\prime}\right)^{-2} \operatorname{sgn}\left(V_{n}\right)_{x} .
\end{aligned}
$$

Integrating the previous equation with respect to $x$ on $\mathbb{R}$ and observing that the terms containing $\left|V_{n}\right| \operatorname{sgn}\left(V_{n}\right)_{x}$ give no contribution to the integral, that $\operatorname{sgn}^{\prime}$ is a positive measure and $b<0$ by assumption (H2), we finally get

$$
\frac{d}{d t} \int_{\mathbb{R}}\left|V_{n}(x, t)\right| d x \leq 0
$$

and therefore,

$$
\int_{\mathbb{R}}\left|V_{n}(x, t)\right| d x \leq \int_{\mathbb{R}}\left|V_{n}(x, 0)\right| d x .
$$

Since $H_{n}(v) \rightarrow H(v)$ pointwisely and $\left|H_{n}(v)-H(v)\right| \leq 2, H_{n}(v) \rightarrow H(v)$ in $L_{\text {loc }}^{1}$. Recalling Lemma 5.1, from (5.6) it follows that

$$
\int_{\mathbb{R}}\left|H(v(x, t))_{x}\right| d x \leq \liminf _{n \rightarrow+\infty} \int_{\mathbb{R}}\left|H_{n}(v(x, t))_{x}\right| d x \leq \liminf _{n \rightarrow+\infty} \int_{\mathbb{R}}\left|H_{n}(v(x, 0))_{x}\right| d x \leq 2 M .
$$

Now, since $H$ is the Heaviside function, $\int_{\mathbb{R}}\left|H(v(x, t))_{x}\right| d x$ gives the number of zeros of $v(x, t)$ where it changes sign and therefore the result.

\section{REFERENCES}

[Bre83] H. Brezis, Analyse Fonctionnelle, Théorie et Applications, Collection Mathématiques Appliqueés pour la Matrise, Masson, Paris, 1983

[Don91] G. C. Dong, Nonlinear partial differential equations of second order, Translations of Mathematical Monographs, Vol. 95, American Mathematical Society, Providence, RI, 1991

[FS98] H. Freistühler and D. Serre, $L^{1}$ Stability of shock waves in scalar viscous conservation laws, Comm. Pure Appl. Math. 51, 291-301 (1998)

[GJK93] R. Gardner, C. K. R. T. Jones, and T. Kapitula, Stability of travelling waves for nonconvex scalar viscous conservation laws, Comm. Pure Appl. Math. 46, 505-526 (1993)

[IO58] A. Ilin and O. Oleinik, Behavior of solutions of the Cauchy problem for certain quasilinear equations for unbounded increase of the time, Dokl. Akad. Nauk SSSR 120, 25-28 (1958) 
[Mar96] P. Marcati, Weak Solutions to a Nonlinear Partial Differential Equation of Mixed Type, Differential Integral Equations 9, 827-848 (1996)

[MN94] A. Matsumura and K. Nishihara, Asymptotic stability of traveling waves for scalar viscous conservation laws with non-convex nonlinearity, Comm. Math. Phys. 165, 83-96 (1994)

[MN96] C. Mascia and R. Natalini, $L^{1}$ Nonlinear stability of travelling waves for a hyperbolic system with relaxation, J. Differential Equations 13, 321-344 (1996)

[OR82] S. Osher and J. Ralston, $L^{1}$ Stability of travelling waves with applications to convective porous media flow, Comm. Pure Appl. Math. 35, 737-751 (1982)

[Ser98] D. Serre, $L^{1}$-Decay and Stability of Shock Profiles, Partial Differential Equations (Praha, 1998), pp. 312-321. Research Notes in Math., vol. 406, Chapman and Hall, Boca Raton, FL., 2000

[Ser99] D. Serre, System of Conservation Laws, Cambridge University Press, Cambridge, 1999

[VH69] A. I. Volpert and S. I. Hudjaev, Cauchy problem for degenerate second order quasilinear parabolic equations, Math. USSR Sbornik 7, 365-387 (1969)

[Wan92] L. Wang, On the regularity theory of fully nonlinear parabolic equations, Comm. Pure Appl. Math. 45, 255-262 (1992) 